\title{
Continuous Non-malleable Codes
}

\author{
Sebastian Faust ${ }^{2}$, Pratyay Mukherjee ${ }^{1}$, \\ Jesper Buus Nielsen ${ }^{1}$, and Daniele Venturi ${ }^{3}$ \\ 1 Department of Computer Science, Aarhus University \\ 2 EPFL Lausanne \\ 3 Department of Computer Science, Sapienza University of Rome
}

\begin{abstract}
Non-malleable codes are a natural relaxation of error correcting/detecting codes that have useful applications in the context of tamper resilient cryptography. Informally, a code is non-malleable if an adversary trying to tamper with an encoding of a given message can only leave it unchanged or modify it to the encoding of a completely unrelated value. This paper introduces an extension of the standard non-malleability security notion - so-called continuous non-malleability - where we allow the adversary to tamper continuously with an encoding. This is in contrast to the standard notion of non-malleable codes where the adversary only is allowed to tamper a single time with an encoding. We show how to construct continuous non-malleable codes in the common split-state model where an encoding consist of two parts and the tampering can be arbitrary but has to be independent with both parts. Our main contributions are outlined below:

1. We propose a new uniqueness requirement of split-state codes which states that it is computationally hard to find two codewords $X=$ $\left(X_{0}, X_{1}\right)$ and $X^{\prime}=\left(X_{0}, X_{1}^{\prime}\right)$ such that both codewords are valid, but $X_{0}$ is the same in both $X$ and $X^{\prime}$. A simple attack shows that uniqueness is necessary to achieve continuous non-malleability in the split-state model. Moreover, we illustrate that none of the existing constructions satisfies our uniqueness property and hence is not secure in the continuous setting.

2. We construct a split-state code satisfying continuous non-malleability. Our scheme is based on the inner product function, collision-resistant hashing and non-interactive zero-knowledge proofs of knowledge and requires an untamperable common reference string.

3. We apply continuous non-malleable codes to protect arbitrary cryptographic primitives against tampering attacks. Previous applications of non-malleable codes in this setting required to perfectly erase the entire memory after each execution and required the adversary to be restricted in memory. We show that continuous non-malleable codes avoid these restrictions.
\end{abstract}

Keywords: non-malleable codes, split-state, tamper resilience.

\section{Introduction}

Physical attacks that target cryptographic implementations instead of breaking the black-box security of the underlying algorithm are amongst the most severe

Y. Lindell (Ed.): TCC 2014, LNCS 8349, pp. 465488 2014.

(C) International Association for Cryptologic Research 2014 
threats for cryptographic systems. A particular important attack on implementations is the so-called tampering attack. In a tampering attack the adversary changes the secret key to some related value and observes the effect of such changes at the output. Traditional black-box security notions do not incorporate adversaries that change the secret key to some related value; even worse, as shown in the celebrated work of Boneh et al. 6] already minor changes to the key suffice for complete security breaches. Unfortunately, tampering attacks are also rather easy to carry out: a virus corrupting a machine can gain partial control over the state, or an adversary that penetrates the cryptographic implementation with physical equipment may induce faults into keys stored in memory.

In recent years, a growing body of work (see [21/22 17/24/1219] and many more) develop new cryptographic techniques that protect against tampering attacks. Non-malleable codes introduced by Dziembowski, Pietrzak and Wichs [17. are an important approach to achieve this goal. Intuitively a code is non-malleable w.r.t. a set of tampering functions $\mathcal{T}$ if the message contained in a codeword modified via a function in $\mathcal{T}$ is either the original message, or a completely unrelated value. Non-malleable codes can be used to protect any cryptographic functionality against tampering with the memory. Instead of storing the key in memory, we store its encoding and decode it each time the functionality wants to accesses the key. As long as the adversary can only apply tampering functions from the set $\mathcal{T}$, the non-malleability property guarantees that the (possibly tampered) decoded value is not related to the original key.

The standard notion of non-malleability considers a one-shot game: the adversary is allowed to tamper a single time with the codeword and obtains the decoded output. In this work we introduce so-called continuous non-malleable codes, where non-malleability is guaranteed even if the adversary continuously applies functions from the set $\mathcal{T}$ to the codeword. We show that our new security notion is not only a natural extension of the standard one-shot notion, but moreover allows to protect against tampering attacks in important settings where earlier constructions fall short to achieve security.

Continuous Non-malleable Codes. A non-malleable code consists of two algorithms Code $=$ (Encode, Decode) that satisfy the correctness property Decode $(\operatorname{Encode}(x))=x$, for all $x \in \mathcal{X}$. To define non-malleability for a function class $\mathcal{T}$, consider the random variable Tamper $_{\mathrm{T}, x}$ defined for every function $\mathrm{T} \in \mathcal{T}$ and any message $x \in \mathcal{X}$ in the game below:

1. Compute an encoding $X \leftarrow \operatorname{Encode}(x)$ using the encoding procedure.

2. Apply the tampering function $T \in \mathcal{T}$ to obtain the tampered codeword $X^{\prime}=\mathrm{T}(X)$.

3. If $X^{\prime}=X$ then return the special symbol same*; otherwise, return $\operatorname{Decode}\left(X^{\prime}\right)$. Notice that $\operatorname{Decode}\left(X^{\prime}\right)$ may return the special symbol $\perp$ in case the tampered codeword $X^{\prime}$ was invalid. 
A coding scheme Code is said to be (one-shot) non-malleable with respect to functions in $\mathcal{T}$ and message space $\mathcal{X}$, if for every $\mathrm{T} \in \mathcal{T}$ and any two messages $x, y \in \mathcal{X}$ the distributions Tamper $_{\mathrm{T}, x}$ and Tamper $_{\mathrm{T}, y}$ are indistinguishable.

To define continuous non-malleable codes, we do not fix a single tampering function T a-priori 1 Instead, we let the adversary repeat step 2 and step 3 from the above game a polynomial number of times, where in each iteration the adversary can adaptively choose a tampering function $\mathrm{T}_{i} \in \mathcal{T}$. We emphasize that this change of the tampering game allows the adversary to tamper continuously with the initial encoding $X$. As shown by Gennaro et al. 21] such a strong security notion is impossible to achieve without further assumptions. To this end, we rely on a self-destruct mechanism as used in earlier works on non-malleable codes. More precisely, when in step 3 the game detects an invalid codeword and returns $\perp$ for the first time, then it self-destructs. This is a rather mild assumption as it can, for instance, be implemented using a single public untamperable bit.

From Non-malleable Codes to Tamper Resilience. As discussed above one main application of non-malleable codes is to protect cryptographic schemes against tampering with the secret key [17/24]. Consider a reactive functionality $\mathcal{G}$ with secret state $s t$ that can be executed on input $m$, e.g., $\mathcal{G}$ may be the AES with key $s t$ encrypting messages $m$. Using a non-malleable code earlier work showed how to transform the functionality $(\mathcal{G}, s t)$ into a functionality $\left(\mathcal{G}^{\text {Code }}, X\right)$ that is secure against tampering with $X$. The transformation compiling $(\mathcal{G}, s t)$ into $\left(\mathcal{G}^{\text {Code }}, X\right)$ works as follows. Initially, $X$ is set to $X \leftarrow \operatorname{Encode}(s t)$. Each time $\mathcal{G}^{\text {Code }}$ is executed on input $m$, the transformed functionality reads the encoding $X$ from memory, decodes it to obtain $s t=\operatorname{Decode}(X)$ and runs the original functionality $\mathcal{G}(s t, m)$. Finally, it erases the memory and stores the new state $X \leftarrow \operatorname{Encode}(s t)$. Additionally to executing evaluation queries the adversary can issue tampering queries $\mathrm{T}_{i} \in \mathcal{T}$. A tampering query replaces the current secret state $X$ with a tampered state $X^{\prime}=\mathrm{T}_{i}(X)$, and the functionality $\mathcal{G}^{\text {Code }}$ continues its computation using $X^{\prime}$ as the secret state. Notice that in case of Decode $\left(X^{\prime}\right)=\perp$ the functionality $\mathcal{G}^{\text {Code }}$ sets the memory to a dummy valueresulting essentially in a self-destruct.

The above transformation guarantees continuous tamper resilience even if the underlying non-malleable code is secure only against one-shot tampering. This security "boost" is achieved by re-encoding the secret state/key after each execution of the primitive $\mathcal{G}^{\text {Code }}$. As one-shot non-malleability suffices in the above cryptographic application, one may ask why we need continuous non-malleable codes. Besides being a natural extension of the standard non-malleability notion, our new notion has several important applications that we discuss in the next two paragraphs.

Tamper Resilience Without Erasures. The transformation described above necessarily requires that after each execution the entire content of the memory is erased. While such perfect erasures may be feasible in some settings, they are

\footnotetext{
${ }^{1}$ Our actual definition is slightly stronger than what is presented next (cf. Section 3).
} 
rather problematic in the presence of tampering. To illustrate this issue consider a setting where besides the encoding of a key, the memory also contains other non-encoded data. In the tampering setting, we cannot restrict the erasure to just the part that stores the encoding of the key as a tampering adversary may copy the encoding to some different part of the memory. A simple solution to this problem is to erase the entire memory, but such an approach is not possible in most cases: for instance, think of the memory as being the hard-disk of your computer that besides the encoding of a key stores other important files that you don't want to be erased. Notice that this situation is quite different from the leakage setting, where we also require perfect erasures to achieve continuous leakage resilience. In the leakage setting, however, the adversary cannot mess around with the state of the memory by, e.g., copying an encoding of a secret key to some free space, which makes erasures significantly easier to implement.

One option to prevent the adversary from keeping permanent copies is to encode the entire state of the memory. Such an approach has, however, the following drawbacks.

1. It is unnatural: In many cases secret data, e.g., a cryptographic key, is stored together with non-confidential data. Each time we want to read some small part of the memory, e.g., the key, we need to decode and re-encode the entire state-including also the non-confidential data.

2. It is inefficient: Decoding and re-encoding the entire state of the memory for each access introduces additional overhead and would result in highly inefficient solutions. This gets even worse as most current constructions of non-malleable codes are rather inefficient.

3. It does not work in general: Consider a setting where we want to compute with non-malleable codes in a tamper resilient way (similar in spirit to tamper resilient circuits). Clearly, in this setting the memory will store many independent encodings of different secrets that cannot be erased. Continuous non-malleable codes are hence a first natural step towards non-malleable computation.

Using our new notion of continuous non-malleable codes we can avoid the above issues and achieve continuous tamper resilience without using erasures and without relying on inefficient solutions that encode the entire state.

Stateless Tamper Resilient Transformations. To achieve tamper resilience from one-shot non-malleability we necessarily need to re-encode the state using fresh randomness. This not only reduces the efficiency of the proposed construction, but moreover makes the transformation stateful. Using continuous non-malleable codes we get continuous tamper resilience for free, eliminating the need to refresh the encoding after each usage. This is in particular useful when the underlying primitive that we want to protect is stateless itself. Think, for instance, of any standard block-cipher construction that typically keeps the same key. Using continuous non-malleable codes the tamper resilient implementation of such stateless primitives does not need to keep any secret state. We discuss the protection of stateless primitives in further detail in Section 5 . 


\subsection{Our Contribution}

In this work, we propose the first construction of continuous non-malleable codes in the split-state model first introduced in the leakage setting [16|13]. Various recent works study the split-state model for non-malleable codes [24|15]1] (see more details on related work in Section 1.2). In the split-state tampering model, the codeword consists of two halves $X_{0}$ and $X_{1}$ that are stored on two different parts of the memory. The adversary is assumed to tamper with both parts independently, but otherwise can apply any efficiently computable tampering function. That is, the adversary picks two polynomial-time computable functions $T_{0}$ and $\mathrm{T}_{1}$ and replaces the state $\left(X_{0}, X_{1}\right)$ with the tampered state $\left(\mathrm{T}_{0}\left(X_{0}\right), \mathrm{T}_{1}\left(X_{1}\right)\right)$. Similar to the earlier work of Liu and Lysyanskaya [24] our construction assumes a public untamperable CRS. Notice that this is a rather mild assumption as the CRS can be hard-wired into the functionality and is independent of any secret data.

Continuous Non-malleability of Existing Constructions. The first construction of (one-shot) split-state non-malleable codes in the standard model was given by Liu and Lysyanskaya [24]. At a high-level the construction encrypts the input $x$ with a leakage resilient encryption scheme and generates a non-interactive zero-knowledge proof of knowledge showing that (a) the public/secret key of the PKE are valid, and (b) the ciphertext is an encryption of $x$ under the public key. Then, $X_{0}$ is set to the secret key while $X_{1}$ holds the corresponding public key, the ciphertext and the above described NIZK proof.

Unfortunately, it is rather easy to break the non-malleable code of Liu and Lysyanskaya in the continuous setting. Recall that our security notion of continuous non-malleable codes allows the adversary to interact in the following game. First, we sample a codeword $\left(X_{0}, X_{1}\right) \leftarrow \operatorname{Encode}(x)$ and then repeat the following process a polynomial number of times:

1. The adversary submits two polynomial-time computable functions $\left(T_{0}, T_{1}\right)$ resulting in a tampered state $\left(X_{0}^{\prime}, X_{1}^{\prime}\right)=\left(\mathrm{T}_{0}\left(X_{0}\right), \mathrm{T}_{1}\left(X_{1}\right)\right)$.

2. We consider three different cases: (1) if $\left(X_{0}^{\prime}, X_{1}^{\prime}\right)=\left(X_{0}, X_{1}\right)$ then return same $^{\star} ;(2)$ otherwise compute $x^{\prime}=\operatorname{Decode}\left(X_{0}^{\prime}, X_{1}^{\prime}\right)$ and return $x^{\prime}$ if $x^{\prime} \neq \perp$; (3) if $x^{\prime}=\perp$ self-destruct and terminate the experiment.

The main observation that enables the attack against the scheme of [24] is as follows. For a fixed (but adversarially chosen) part $X_{0}^{\prime}$ it is easy to come-up with two corresponding parts $X_{1}^{\prime}$ and $X_{1}^{\prime \prime}$ such that both $\left(X_{0}^{\prime}, X_{1}^{\prime}\right)$ and $\left(X_{0}^{\prime}, X_{1}^{\prime \prime}\right)$ form a valid codeword that does not lead to a self-destruct. Suppose further that $\operatorname{Decode}\left(X_{0}^{\prime}, X_{1}^{\prime}\right) \neq \operatorname{Decode}\left(X_{0}^{\prime}, X_{1}^{\prime \prime}\right)$, then under continuous tampering the adversary may permanently replace the original encoding $X_{0}$ with $X_{0}^{\prime}$, while depending on whether the $i$-th bit of $X_{1}$ is 0 or 1 either replace $X_{1}$ by $X_{1}^{\prime}$ or $X_{1}^{\prime \prime}$. This allows to recover the entire $X_{1}$ by just $\left|X_{1}\right|$ tampering attacks. Once $X_{1}$ is known to the adversary it is easy to tamper with $\left(X_{0}, X_{1}\right)$ in a way that depends on Decode $\left(X_{0}, X_{1}\right)$.

Somewhat surprisingly, our attack can be generalized to break any nonmalleable code that is secure in the information theoretic setting. Hence, also 
the recent breakthrough results on information theoretic non-malleability [15]1] fail to provide security under continuous attacks. Moreover, we emphasize that our attack does not only work for the code itself, but (in most cases) can be also applied to the tamper-protection application of cryptographic functionalities.

Uniqueness. The attack above exploits that for a fixed known part $X_{0}^{\prime}$ it is easy to come-up with two valid parts $X_{1}^{\prime}, X_{1}^{\prime \prime}$. For the encoding of [24] this is indeed easy to achieve. If the secret key $X_{0}^{\prime}$ is known it is easy to come-up with two valid parts $X_{1}^{\prime}, X_{1}^{\prime \prime}$ : just encrypt two arbitrary messages $x_{0} \neq x_{1}$ and generate the corresponding proofs. The above weakness motivates a new property that non-malleable codes shall satisfy in order to achieve security against continuous non-malleability. We call this property uniqueness, which informally guarantees that for any (adversarially chosen) valid encoding $\left(X_{0}^{\prime}, X_{1}^{\prime}\right)$ it is computationally hard to come up with $X_{b}^{\prime \prime} \neq X_{b}^{\prime}$ such that $\left(X_{b}^{\prime}, X_{1-b}^{\prime \prime}\right)$ forms a valid encoding. Clearly the uniqueness property prevents the above described attack, and hence is a crucial requirement for continuous non-malleability.

A New Construction. In light of the above discussion, we need to build a nonmalleable code that achieves our uniqueness property. Our construction uses as building blocks a leakage resilient storage (LRS) scheme [13]14 for the split-state model (one may view this as a generalization of the leakage resilient PKE used in 24]), a collision-resistant hash function and (similar to [24]) an extractable NIZK. At a high-level we use the LRS to encode the secret message, hash the resulting shares using the hash function and generate a NIZK proof of knowledge that indeed the resulting hash values are correctly computed from the shares. While it is easy to show that collision resistance of the hash function guarantees the uniqueness property, a careful analysis is required to prove continuous nonmalleability. We refer the reader to Section 4 for the details of our construction and to Section 4.1 for an outline of the proof.

Tamper Resilience for Stateless and Stateful Primitives. We can use our new construction of continuous non-malleable codes to protect arbitrary computation against continuous tampering attacks. In contrast to earlier works our construction does not need to re-encode the secret state after each usage, which besides being more efficient avoids the use of erasures. As discussed above, erasures are problematic in the tampering setting as one would essentially need to encode the entire state (possibly including large non-confidential data).

Additionally, our transformation does not need to keep any secret state. Hence, if our transformation is used for stateless primitives, then the resulting scheme remains stateless. This solves an open problem of Dziembowski, Pietrzak and Wichs [17. Notice that while we do not need to keep any secret state, the transformed functionality requires one single bit to switch to self-destruction mode. This bit can be public but must be untamperable, and can for instance be implemented through one-time writable memory. As shown in the work of Gennaro et al. [21] continuous tamper resilience is impossible to achieve without such a mechanism for self-destruction. 
Of course, our construction can also be used for stateful primitives, in which case our functionality will re-encode the new state during execution. Note that in this setting, as data is never erased, an adversary can always reset the functionality to a previous valid state. To avoid this, our transformation uses an untamperable public counter 2 that helps us to detect whenever the functionality is reset to a previous state, leading to a self-destruct. We notice that such an untamperable counter is necessary, as otherwise there is no way to protect against the above resetting attack.

Adding Leakage. As a last contribution, we show that our code is also secure against bounded leakage attacks. This is similar to the works of [24]15] who also consider bounded leakage resilience of their encoding scheme. We then show that bounded leakage resilience is also inherited by functionalities that are protected by our transformation. Notice that without perfect erasures bounded leakage resilience is the best we can achieve, as there is no hope for security if an encoding that is produced at some point in time is gradually revealed to the adversary.

\subsection{Related Work}

Constructions of Non-malleable Codes. Besides showing feasibility by a probabilistic argument, [17] also built non-malleable codes for bit-wise tampering and gave a construction in the split-state model using a random oracle. This result was followed by [9] which proposed non-malleable codes that are secure against block-wise tampering. The first construction of non-malleable codes in the splitstate model was given by Liu and Lysyanskaya [24] assuming an untamperable CRS. Very recently two beautiful works showed how to build non-malleable codes in the split-state model without relying on a CRS [151] even when the adversary has unlimited computing power. Dziembowski et al. [15] show how to encode a single bit using the inner product function. Agrawal et al. [1] developed a construction that goes beyond single-bit encoding but induces a huge overhead.

See also [8/719] for other recent advances on the construction of non-malleable codes. We also notice that the work of Genarro et al. 21. proposed a generic method that allows to protect arbitrary computation against continuous tampering attacks, without requiring erasures. We refer the reader to [17] for a more detailed comparison between non-malleable codes and the solution of [21].

Other Works on Tamper Resilience. A large body of work shows how to protect specific cryptographic schemes against tampering attacks (see [432352512 and many more). While these works consider a strong tampering model (e.g., they do not require the split-state assumption), they only offer security for specific schemes. In contrast non-malleable codes are generally applicable and can provide tamper resilience of any cryptographic scheme.

In all the above works, including ours, it is assumed that the circuitry that computes the cryptographic algorithm using the potentially tampered key runs

\footnotetext{
${ }^{2}$ Note that a counter uses very small (logarithmic in the security parameter) number of bits.
} 
correctly, and is not subject to tampering attacks. An important line of works analyze to what extent we can guarantee security when even the circuitry is prone to tampering attacks 222011. These works typically consider a restricted class of tampering attacks (e.g., individual bit tampering) and assume that large parts of the circuit (and memory) remain untampered.

\section{Preliminaries}

\subsection{Notation}

We let $\mathbb{N}$ be the set of naturals. For $n \in \mathbb{N}$, we write $[n]:=\{1, \ldots, n\}$. Given a set $\mathcal{S}$, we write $s \leftarrow \mathcal{S}$ to denote that element $s$ is sampled uniformly from $\mathcal{S}$. If $\mathrm{S}$ is an algorithm, $y \leftarrow \mathrm{S}(x)$ denotes an execution of $\mathrm{S}$ with input $x$ and output $y$; if $\mathrm{S}$ is randomized, then $y$ is a random variable.

Throughout the paper we denote the security parameter by $k \in \mathbb{N}$. A function $\delta(k)$ is called negligible in $k$ (or simply negligible) if it vanishes faster than the inverse of any polynomial in $k$, i.e., $\delta(k)=k^{-\omega(1)}$. A machine $\mathrm{S}$ is called probabilistic polynomial time (PPT) if for any input $x \in\{0,1\}^{*}$ the computation of $\mathrm{S}(x)$ terminates in at most poly $(|x|)$ steps and $\mathrm{S}$ is probabilistic (i.e., it uses randomness as part of its logic).

Oracle $\mathcal{O}^{\ell}(s)$ is parametrized by a value $s$ and takes as input functions $\mathrm{L}$ and outputs $\mathrm{L}(s)$, returning a total of at most $\ell$ bits.

\subsection{Robust Non-interactive Zero Knowledge}

Given an NP-relation, let $\mathcal{L}=\{x: \exists w$ such that $\mathcal{R}(x, w)=1\}$ be the corresponding language. A robust non-interactive zero knowledge (NIZK) proof system for $\mathcal{L}$, is a tuple of algorithms $\left(\mathrm{G}_{\mathrm{NIZK}}\right.$, Prove, Verify, $\left.\operatorname{Sim}=\left(\mathrm{Sim}_{1}, \mathrm{Sim}_{2}\right), \mathrm{Xtr}\right)$ such that the following properties hold [26].

Completeness. For all $x \in \mathcal{L}$ of length $k$ and all $w$ such that $\mathcal{R}(x, w)=1$, for all $\Omega \leftarrow \operatorname{G}_{\mathrm{NIZK}}\left(1^{k}\right)$ we have that $\operatorname{Verify}(\Omega, x$, Prove $(\Omega, w, x))=$ accept

Multi-theorem zero knowledge. For all PPT adversaries $\mathrm{A}$, we have $\operatorname{Real}(k) \approx$ $\operatorname{Simu}(\mathrm{k})$, where $\operatorname{Real}(k)$ and $\operatorname{Simu}(\mathrm{k})$ are distributions defined via the following experiment:

$$
\begin{aligned}
\operatorname{Real}(k) & =\left\{\Omega \leftarrow \mathrm{G}_{\mathrm{NIZK}}\left(1^{k}\right) ; \text { out } \leftarrow \mathrm{A}^{\operatorname{Prove}(\Omega, \cdot, \cdot)}(\Omega) ; \text { Output: out. }\right\} \\
\operatorname{Simu}(k) & =\left\{(\Omega, t k) \leftarrow \operatorname{Sim}_{1}\left(1^{k}\right) ; \text { out } \leftarrow \mathrm{A}^{\operatorname{Sim}_{2}(\Omega, \cdot, t k)}(\Omega) ; \text { Output: out. }\right\} .
\end{aligned}
$$

Extractability. There exists a PPT algorithm Xtr such that, for all PPT adversaries $A$, we have

$$
\mathbb{P}\left[\begin{array}{c}
(\Omega, t k, e k) \leftarrow \operatorname{Sim}_{1}\left(1^{k}\right) ;(x, \pi) \leftarrow \mathrm{A}^{\operatorname{Sim}_{2}(\Omega, \cdot, t k)}(\Omega) ; \\
w \leftarrow \operatorname{Xtr}(\Omega,(x, \pi), e k) \\
\mathcal{R}(x, w) \neq 1 \wedge(x, \pi) \notin \mathcal{Q} \wedge \operatorname{Verify}(\Omega, x, \pi)=\text { accept }
\end{array}\right] \leq n e g l(k),
$$

where the list $\mathcal{Q}$ contains the successful pairs $\left(x_{i}, \pi_{i}\right)$ that $\mathrm{A}$ has queried to $\mathrm{Sim}_{2}$. 
Similarly to 24], we assume that different statements have different proofs, i.e., if $\operatorname{Verify}(\Omega, x, \pi)=$ accept we have that $\operatorname{Verify}\left(\Omega, x^{\prime}, \pi\right)=$ reject for all $x^{\prime} \neq x$. This property can be achieved by appending the statement to its proof.

We also require that the proof system supports labels, so that the Prove, Verify, Sim and Xtr algorithms now also take a public label $\lambda$ as input, and the completeness, zero knowledge and extractability properties are updated accordingly. (This can be easily achieved by appending the label $\lambda$ to the statement $x$.) More precisely, we write $\operatorname{Prove}^{\lambda}(\Omega, w, x)$ and $\operatorname{Verify}^{\lambda}(\Omega, x, \pi)$ for the prover and the verifier, and $\operatorname{Sim}_{2}^{\lambda}(\Omega, x, t k)$ and $\operatorname{Xtr}^{\lambda}(\Omega,(x, \pi), e k)$ for the simulator and the extractor.

\subsection{Leakage Resilient Storage}

We recall the definition of leakage resilient storage from [1314]. A leakage resilient storage scheme (LRS, LRS ${ }^{-1}$ ) is a pair of algorithms defined as follows. (1) Algorithm LRS takes as input a secret $x$ and outputs an encoding $\left(s_{0}, s_{1}\right)$ of $x$. (2) Algorithm $\mathrm{LRS}^{-1}$ takes as input shares $\left(s_{0}, s_{1}\right)$ and outputs a message $x^{\prime}$. Since the LRS that we use in this paper is secure against computationally unbounded adversaries, we state the definition below in the information theoretic setting. It is easy to extend it to also consider computationally bounded adversaries.

Definition 1 (LRS). We call $\left(\mathrm{LRS}_{\mathrm{LRS}}{ }^{-1}\right)$ an $\ell$-leakage resilient storage scheme $(\ell-L R S)$ if for all $\theta \in\{0,1\}$, all secrets $x, y$ and all adversaries $A$ it holds that

$$
\left\{\text { Leakage }_{\mathrm{A}, x, \theta}(k)\right\}_{k \in \mathbb{N}} \approx_{s}\left\{\text { Leakage }_{\mathrm{A}, y, \theta}(k)\right\}_{k \in \mathbb{N}},
$$

where

$$
\text { Leakage }_{\mathrm{A}, x, \theta}(k)=\left\{\begin{array}{c}
\left(s_{0}, s_{1}\right) \leftarrow \operatorname{LRS}(x) ; \text { out }_{\mathrm{A}} \leftarrow \mathrm{A}^{\mathcal{O}^{\ell}\left(s_{0}, \cdot\right), \mathcal{O}^{\ell}\left(s_{1}, \cdot\right)} ; \\
\text { Output: }\left(s_{\theta}, \text { out }_{\mathrm{A}}\right) .
\end{array}\right\} .
$$

We remark that Definition 1 is stronger than the standard definition of LRS, in that the adversary is allowed to see one of the two shares after he is done with leakage queries. A careful analysis of the proof, however, shows that the LRS scheme of [14, Lemma 22] satisfies the above generalized notion since the inner product function is a strong randomness extractor [10].

\section{Continuous Non-malleability}

We start by formally defining an encoding scheme in the common reference string (CRS) model.

Definition 2 (Split-state Encoding Scheme in the CRS Model). A splitstate encoding scheme in the common reference string (CRS) model is a tuple of algorithms Code $=$ (Init, Encode, Decode) specified below.

- Init takes as input the security parameter and outputs a CRS $\Omega \leftarrow \operatorname{Init}\left(1^{k}\right)$. 
- Encode takes as input some message $x \in\{0,1\}^{k}$ and the CRS $\Omega$ and outputs a codeword consisting of two parts $\left(X_{0}, X_{1}\right)$ such that $X_{0}, X_{1} \in\{0,1\}^{n}$.

- Decode takes as input a codeword $\left(X_{0}, X_{1}\right) \in\{0,1\}^{2 n}$ and the CRS and outputs either a message $x^{\prime} \in\{0,1\}^{k}$ or a special symbol $\perp$.

Consider the following oracle $\mathcal{O}_{\mathrm{cnm}}\left(\left(X_{0}, X_{1}\right)\right)$, which is parametrized by an encoding $\left(X_{0}, X_{1}\right)$ and takes as input functions $\mathrm{T}_{0}, \mathrm{~T}_{1}:\{0,1\}^{n} \rightarrow\{0,1\}^{n}$.

$$
\begin{aligned}
& \frac{\mathcal{O}_{\mathrm{cnm}}\left(\left(X_{0}, X_{1}\right),\left(\mathrm{T}_{0}, \mathrm{~T}_{1}\right)\right):}{}\left(X_{0}^{\prime}, X_{1}^{\prime}\right)=\left(\mathrm{T}_{0}\left(X_{0}\right), \mathrm{T}_{1}\left(X_{1}\right)\right) \\
& \quad \text { If }\left(X_{0}^{\prime}, X_{1}^{\prime}\right)=\left(X_{0}, X_{1}\right) \text { return same* } \\
& \quad \text { If Decode }\left(\Omega,\left(X_{0}^{\prime}, X_{1}^{\prime}\right)\right)=\perp, \text { return } \perp \text { and "self-destruct" } \\
& \quad \text { Else return }\left(X_{0}^{\prime}, X_{1}^{\prime}\right) .
\end{aligned}
$$

By "self-destruct" we mean that once Decode $\left(\Omega,\left(X_{0}^{\prime}, X_{1}^{\prime}\right)\right)$ outputs $\perp$, the oracle will answer $\perp$ to any further query.

Definition 3 (Continuous Non-Malleability). Let Code = (Init, Encode, Decode) be a split-state encoding scheme in the CRS model. We say that Code is q-continuously non-malleable $\ell$-leakage resilient $((\ell, q)$-CNMLR for short), if for all messages $x, y \in\{0,1\}^{k}$ and all PPT adversaries $\mathrm{A}$ it holds that

$$
\left\{\operatorname{Tamper}_{\mathrm{A}, x}^{\mathrm{cnmlr}}(k)\right\}_{k \in \mathbb{N}} \approx_{c}\left\{\operatorname{Tamper}_{\mathrm{A}, y}^{\mathrm{cnmlr}}(k)\right\}_{k \in \mathbb{N}}
$$

where

$$
\operatorname{Tamper}_{\mathrm{A}, x}^{\mathrm{cnmlr}}(k)=\left\{\begin{array}{c}
\Omega \leftarrow \operatorname{Init}\left(1^{k}\right) ;\left(X_{0}, X_{1}\right) \leftarrow \operatorname{Encode}(\Omega, x) ; \\
\text { out }_{\mathrm{A}} \leftarrow \mathrm{A}^{\mathcal{O}^{\ell}\left(X_{0}\right), \mathcal{O}^{\ell}\left(X_{1}\right), \mathcal{O}_{\mathrm{cnm}}\left(\left(X_{0}, X_{1}\right)\right)} ; \text { Output: out } \\
\mathrm{A}
\end{array}\right\}
$$

and $\mathrm{A}$ asks a total of $q$ queries to $\mathcal{O}_{\mathrm{cnm}}$.

Without loss of generality we assume that the variable out $_{A}$ consists of all the bits leaked from $X_{0}$ and $X_{1}$ (in a vector $\boldsymbol{\Lambda}$ ) and all the outcomes from oracle $\mathcal{O}_{\mathrm{cnm}}\left(X_{0}, X_{1}\right)$ (in a vector $\left.\boldsymbol{\Theta}\right)$; we write this as out $A=(\boldsymbol{\Lambda}, \boldsymbol{\Theta})$ where $|\boldsymbol{\Lambda}| \leq 2 \ell$ and $\boldsymbol{\Theta}$ has exactly $q$ elements.

Intuitively, the above definition captures a setting where a fully adaptive adversary $A$ tries to break non-malleability by tampering several times with a target encoding, obtaining each time some leakage from the decoding process. The only restriction is that whenever a tampering attempt decodes to $\perp$, the system "selfdestructs" 3 Note that whenever the adversary mauls $\left(X_{0}, X_{1}\right)$ to a valid encoding $\left(X_{0}^{\prime}, X_{1}^{\prime}\right)$, oracle $\mathcal{O}_{\mathrm{cnm}}$ returns $\left(X_{0}^{\prime}, X_{1}^{\prime}\right)$. This is different from [1724], where the experiment returns the output of the decoded message, i.e. Decode $\left(\Omega,\left(X_{0}^{\prime}, X_{1}^{\prime}\right)\right)$. The recent work of Faust et al. [19] consider a similar extension where also the codeword is returned instead of the decoded message and call it super strong nonmalleability. Also, we remark that Definition 3 implies strong non-malleability

\footnotetext{
${ }^{3}$ As described in 21 it is easy to see that without such a restriction non-malleability can indeed be broken, since $\mathrm{A}$ can simply recover the entire $\left(X_{0}, X_{1}\right)$ after polynomially many queries.
} 
(as defined in [17 24]) if we restrict $\mathrm{A}$ to ask a single query (i.e., $q=1$ ) to oracle $\mathcal{O}_{\text {cnm }} 4$ We choose the formulation above because it is stronger and at the same time achieved by our code!

\subsection{Uniqueness}

As we argue below, constructions that satisfy our new Definition 3 have to meet the following uniqueness requirement. Informally this means that for any (possibly adversarially chosen) side of an encoding $X_{b}^{\prime}$ it is computationally hard to find two corresponding sides $X_{1-b}^{\prime}$ and $X_{1-b}^{\prime \prime}$ such that both $\left(X_{b}^{\prime}, X_{1-b}^{\prime}\right)$ and $\left(X_{b}^{\prime}, X_{1-b}^{\prime \prime}\right)$ form a valid encoding.

Definition 4 (Uniqueness). Let Code = (Init, Encode, Decode) be a split-state encoding in the CRS model. We say that Code satisfies uniqueness if for all PPT adversaries $\mathrm{A}$ and for all $b \in\{0,1\}$ we have:

$$
\mathbb{P}\left[\begin{array}{c}
\Omega \leftarrow \operatorname{Init}\left(1^{k}\right) ;\left(X_{b}^{\prime}, X_{1-b}^{\prime}, X_{1-b}^{\prime \prime}\right) \leftarrow \mathrm{A}\left(1^{k}, \Omega\right) ; X_{1-b}^{\prime} \neq X_{1-b}^{\prime \prime} ; \\
\operatorname{Decode}\left(\Omega,\left(X_{b}^{\prime}, X_{1-b}^{\prime}\right)\right) \neq \perp ; \operatorname{Decode}\left(\Omega,\left(X_{b}^{\prime}, X_{1-b}^{\prime \prime}\right)\right) \neq \perp
\end{array}\right] \leq \operatorname{negl}(k) .
$$

The following attack shows that the uniqueness property is necessary to achieve Definition 3 .

Lemma 1. Let Code be $(0$, poly $(k))$-CNMLR. Then Code must satisfy uniqueness.

Proof. For the sake of contradiction, assume that we can efficiently find a triple $\left(X_{0}^{\prime}, X_{1}^{\prime}, X_{1}^{\prime \prime}\right)$ such that $\left(X_{0}^{\prime}, X_{1}^{\prime}\right)$ and $\left(X_{0}^{\prime}, X_{1}^{\prime \prime}\right)$ are both valid and $X_{1}^{\prime} \neq X_{1}^{\prime \prime}$. For a target encoding $\left(Y_{0}, Y_{1}\right)$, we describe an efficient algorithm recovering $Y_{1}$ with overwhelming probability, by asking $n=\operatorname{poly}(k)$ queries to $\mathcal{O}_{\mathrm{cnm}}\left(\left(Y_{0}, Y_{1}\right), \cdot\right)$.

For all $i \in[n]$ repeat the following:

Prepare the $i$-th tampering function as follows:

- $\mathrm{T}_{0}^{(i)}\left(Y_{0}\right)$ : Replace $Y_{0}$ by $X_{0}^{\prime}$;

- $\mathrm{T}_{1}^{(i)}\left(Y_{1}\right)$ : If $Y_{1}[i]=0$ replace $Y_{1}$ by $X_{1}^{\prime}$; otherwise replace it by $X_{1}^{\prime \prime}$.

Submit $\left(\mathrm{T}_{0}^{(i)}, \mathrm{T}_{1}^{(i)}\right)$ to $\mathcal{O}_{\mathrm{cnm}}\left(\left(Y_{0}, Y_{1}\right), \cdot\right)$ and obtain $\left(Y_{0}^{\prime}, Y_{1}^{\prime}\right)$.

If $\left(Y_{0}^{\prime}, Y_{1}^{\prime}\right)=\left(X_{0}^{\prime}, X_{1}^{\prime}\right)$, set $Z[i] \leftarrow 0$.

Otherwise, if $\left(Y_{0}^{\prime}, Y_{1}^{\prime}\right)=\left(X_{0}^{\prime}, X_{1}^{\prime \prime}\right)$, set $Z[i] \leftarrow 1$.

Output $Z$ as the guess for $Y_{1}$.

The above algorithm clearly succeeds with overwhelming probability, whenever $X_{1}^{\prime} \neq Y_{1} \neq X_{1}^{\prime \prime} 5$

${ }^{4}$ It is easy to see that encoding from [24] satisfies the stronger variant of strong nonmalleability.

${ }^{5}$ In case $\left(X_{0}^{\prime}, X_{1}^{\prime}\right)=\left(Y_{0}, Y_{1}\right)$ or $\left(X_{0}^{\prime}, X_{1}^{\prime \prime}\right)=\left(Y_{0}, Y_{1}\right)$, then the entire encoding can be recovered even with more ease. In this case, whenever the oracle returns same ${ }^{\star}$ we know $Y_{0}=X_{0}^{\prime}$ and $Y_{1} \in\left\{X_{1}^{\prime}, X_{1}^{\prime \prime}\right\}$. In the next step we replace the encoding with $\left(X_{0}^{\prime}, X_{1}^{\prime}\right)$; if the oracle returns same ${ }^{\star}$ again, then we conclude that $Y_{1}=X_{1}^{\prime}$, otherwise we conclude $Y_{1}=X_{1}^{\prime \prime}$. 
Once $Y_{1}$ is known, we ask one additional query $\left(\mathrm{T}_{0}^{(n+1)}, \mathrm{T}_{1}^{(n+1)}\right)$ to $\mathcal{O}_{\mathrm{cnm}}\left(\left(Y_{0}, Y_{1}\right), \cdot\right)$, as follows:

- $\mathrm{T}_{0}^{(n+1)}\left(Y_{0}\right)$ hard-wires $Y_{1}$ and computes $y \leftarrow \operatorname{Decode}\left(\Omega,\left(Y_{0}, Y_{1}\right)\right)$; if the first bit of $y$ is 0 then $\mathrm{T}_{0}$ behaves like the identity function, otherwise it overwrites $Y_{0}$ with $0^{n}$.

$-\mathrm{T}_{1}^{(n+1)}\left(Y_{0}\right)$ is the identity function.

The above clearly allows to learn one bit of the message in the target encoding and hence contradicts the fact that Code is $(0$, poly $(k))$-CNMLR.

Attacking existing schemes. The above procedure can be applied to show that the encoding of [24] does not satisfy our notion. Recall that in 24] a message $x$ is encoded as $X_{0}=(p k, c:=\operatorname{Enc}(p k, x), \pi)$ and $X_{1}=s k$. Here, $(p k, s k)$ is a valid key pair and $\pi$ is a proof of knowledge of a pair $(x, s k)$ such that $c$ decrypts to $x$ under $s k$ and $(p k, s k)$ forms a valid key-pair. Clearly, for some $X_{1}^{\prime}=s k^{\prime}$ it is easy to find two valid corresponding parts $X_{0}^{\prime} \neq X_{0}^{\prime \prime}$ which violates uniqueness.

We mention two important extensions of the attack from Lemma 1) leading to even stronger security breaches:

1. In case the valid pair of encodings $\left(X_{0}^{\prime}, X_{1}^{\prime}\right),\left(X_{0}^{\prime}, X_{1}^{\prime \prime}\right)$ which violates the uniqueness property are such that $\operatorname{Decode}\left(\Omega,\left(X_{0}^{\prime}, X_{1}^{\prime}\right)\right) \neq$ $\operatorname{Decode}\left(\Omega,\left(X_{0}^{\prime}, X_{1}^{\prime \prime}\right)\right)$, one can show that Lemma 1 still holds in the weaker version of the Definition 3 in which the experiment does not output tampered encodings but only the corresponding decoded message. Note that this applies in particular to the encoding of [24].

2. In case it is possible to find both $\left(X_{0}^{\prime}, X_{1}^{\prime}, X_{1}^{\prime \prime}\right)$ and $\left(X_{0}^{\prime}, X_{0}^{\prime \prime}, X_{1}^{\prime}\right)$ violating uniqueness, a simple variant of the attack allows us to recover both halves of the target encoding which is a total breach of security! However, it is not clear for the scheme of [24] how to find two valid corresponding parts $X_{1}^{\prime}, X_{1}^{\prime \prime}$, because given $p k^{\prime}$ it shall of course be computationally hard to find two corresponding valid secret keys $s k^{\prime}, s k^{\prime \prime}$.

The above attack can be easily extended to the information theoretic setting to break the constructions of the non-malleable codes (in split-state) recently introduced in 15] and in [1. In fact, in the following lemma we show that there does not exist any information theoretic secure CNMLR code.

Lemma 2. It is impossible to construct information theoretically secure $(0$, poly $(k))-C N M L R$ codes.

Proof. We prove the lemma by contradiction. Assume that there exists an information theoretically secure $(0$, poly $(k))$-CNMLR code with $2 n$ bits codewords. By Lemma 1, the code must satisfy the uniqueness property. In the information theoretic setting this means that, for all codewords $\left(X_{0}, X_{1}\right) \in\{0,1\}^{2 n}$ such that $\operatorname{Decode}\left(\Omega,\left(X_{0}, X_{1}\right)\right) \neq \perp$, the following holds: (i) for all $X_{1}^{\prime} \in\{0,1\}^{n}$ such that $X_{1}^{\prime} \neq X_{1}$, we have $\operatorname{Decode}\left(\Omega,\left(X_{0}, X_{1}^{\prime}\right)\right)=\perp$; (ii) for all $X_{0}^{\prime} \in\{0,1\}^{n}$, such that $X_{0}^{\prime} \neq X_{0}$, we have $\operatorname{Decode}\left(\Omega,\left(X_{0}^{\prime}, X_{1}\right)\right)=\perp$. 
Given a target encoding $\left(X_{0}, X_{1}\right)$ of some secret $x$, an unbounded A can define the following tampering function $\mathrm{T}_{b}$ (for $b \in\{0,1\}$ ): Given $X_{b}$ as input, try all possible $X_{1-b} \in\{0,1\}^{n}$ until $\operatorname{Decode}\left(\Omega,\left(X_{0}, X_{1}\right)\right) \neq \perp$. By property (i)-(ii) above, we conclude that for all $X_{1-b}^{\prime} \neq X_{1-b}$, the decoding algorithm $\operatorname{Decode}\left(\Omega,\left(X_{b}, X_{1-b}^{\prime}\right)\right)$ outputs $\perp$ with overwhelming probability. Thus, $\mathrm{T}_{b}$ can recover $x=\operatorname{Decode}\left(\Omega,\left(X_{b}, X_{1-b}\right)\right)$ and if the first bit of the decoded value is 0 leave the target encoding unchanged, otherwise $\left(T_{0}, T_{1}\right)$ modifies the encoding with an invalid codeword. The above clearly allows to learn one bit of the message in the target encoding, and hence contradicts the fact that the code is $(0, \operatorname{poly}(k))$-CNMLR.

Note that the attack of Lemma 2 requires the tampering function to be unbounded. In case when the tampering functions are computationally bounded and only the adversary is computationally unbounded we do not know how to make the above attack work.

\section{The Code}

Consider the following split-state encoding scheme in the CRS model (Init, Encode, Decode), based on an LRS scheme (LRS, $\mathrm{LRS}^{-1}$ ), on a family of collision resistant hash functions $\mathcal{H}=\left\{H_{t}:\{0,1\}^{\text {poly }(k)} \rightarrow\{0,1\}^{k}\right\}_{t \in\{0,1\}^{k}}$ and on a robust non-interactive zero knowledge proof system $\left(\mathrm{G}_{\mathrm{NIZK}}\right.$, Prove, Verify) which supports labels, for language $\mathcal{L}_{\mathcal{H}, t}=\left\{h: \exists s\right.$ such that $\left.h=H_{t}(s)\right\}$.

$\operatorname{Init}\left(1^{k}\right)$. Sample $t \leftarrow\{0,1\}^{k}$ and run $\Omega \leftarrow \mathrm{G}_{\mathrm{NIZK}}\left(1^{k}\right)$.

Encode $(\Omega, x)$. Let $\left(s_{0}, s_{1}\right) \leftarrow \operatorname{LRS}(x)$. Compute $h_{0}=H_{t}\left(s_{0}\right), h_{1}=H_{t}\left(s_{1}\right)$ and $\pi_{0} \leftarrow \operatorname{Prove}^{\lambda_{1}}\left(\Omega, s_{0}, h_{0}\right), \pi_{1} \leftarrow \operatorname{Prove}^{\lambda_{0}}\left(\Omega, s_{1}, h_{1}\right)$, where the labels are defined as $\lambda_{0}=h_{0}, \lambda_{1}=h_{1}$. (Note that the pre-image of $h_{b}$ is $s_{b}$ and the proof $\pi_{b}$ is computed for statement $h_{b}$ using label $h_{1-b}$.) Output $\left(X_{0}, X_{1}\right)=\left(\left(s_{0}, h_{1}, \pi_{1}, \pi_{0}\right),\left(s_{1}, h_{0}, \pi_{0}, \pi_{1}\right)\right)$.

$\operatorname{Decode}\left(\Omega,\left(X_{0}, X_{1}\right)\right)$. The decoding parses $X_{b}$ as $\left(s_{b}, h_{1-b}, \pi_{1-b}, \pi_{b}\right)$, computes $\lambda_{b}=H_{t}\left(s_{b}\right)$ and then proceeds as follows:

(a) Local check. If Verify ${ }^{\lambda_{1}}\left(\Omega, h_{0}, \pi_{0}\right)$ or $\operatorname{Verify}^{\lambda_{0}}\left(\Omega, h_{1}, \pi_{1}\right)$ output reject in any of the two sides $X_{0}, X_{1}$, return $x^{\prime}=\perp$.

(b) Cross check. If (i) $h_{0} \neq H_{t}\left(s_{0}\right)$ or $h_{1} \neq H_{t}\left(s_{1}\right)$, or (ii) the proofs $\left(\pi_{0}, \pi_{1}\right)$ in $X_{0}$ are different from the ones in $X_{1}$, then return $x^{\prime}=\perp$.

(c) Decoding. Otherwise, return $x^{\prime}=\operatorname{LRS}^{-1}\left(s_{0}, s_{1}\right)$.

We start by showing that the above code satisfies the uniqueness property (cf. Definition 4).

Lemma 3. Let Code $=$ (Init, Encode, Decode) be as above. Then, if $\mathcal{H}$ is a family of collision resistant hash functions Code satisfies uniqueness.

Proof. We show that Definition 4 is satisfied for $b=0$. The proof for $b=1$ is identical and is therefore omitted. 
Assume that there exists a PPT adversary A that, given as input $\Omega \leftarrow$ $\operatorname{Init}\left(1^{k}\right)$, is able to produce $\left(X_{0}^{\prime}, X_{1}^{\prime}, X_{1}^{\prime \prime}\right)$ such that both $\left(X_{0}^{\prime}, X_{1}^{\prime}\right)$ and $\left(X_{0}^{\prime}, X_{1}^{\prime \prime}\right)$ are valid, but $X_{1}^{\prime} \neq X_{1}^{\prime \prime}$. Let $X_{0}^{\prime}=\left(s_{0}^{\prime}, h_{1}^{\prime}, \pi_{1}^{\prime}, \pi_{0}^{\prime}\right), X_{1}^{\prime}=\left(s_{1}^{\prime}, h_{0}^{\prime}, \pi_{0}^{\prime}, \pi_{1}^{\prime}\right)$ and $X_{1}^{\prime \prime}=\left(s_{1}^{\prime \prime}, h_{0}^{\prime \prime}, \pi_{0}^{\prime \prime}, \pi_{1}^{\prime \prime}\right)$.

Since $s_{0}^{\prime}$ is the same in both encodings, we must have $h_{0}^{\prime}=h_{0}^{\prime \prime}$ as the hash function is deterministic. Furthermore, since both $\left(X_{0}^{\prime}, X_{1}^{\prime}\right)$ and $\left(X_{0}^{\prime}, X_{1}^{\prime \prime}\right)$ are valid, the proofs must verify successfully and therefore we must have $\pi_{0}^{\prime}=\pi_{0}^{\prime \prime}$ and $\pi_{1}^{\prime}=\pi_{1}^{\prime \prime}$. It follows that $X_{1}^{\prime \prime}=\left(s_{1}^{\prime \prime}, h_{0}^{\prime}, \pi_{0}^{\prime}, \pi_{1}^{\prime}\right)$, such that $s_{1}^{\prime \prime} \neq s_{1}^{\prime}$. Clearly $\left(s_{1}^{\prime}, s_{1}^{\prime \prime}\right)$ is a collision for $h_{1}^{\prime}$, a contradiction.

While the uniqueness property is a necessary requirement to achieve continuous non-malleability, showing that that the above code is a continuous non-malleable and leakage resilient code requires to overcome several technical challenges. We next state our main theorem and give a proof outline in the following section. The full proof of Theorem [1 is deferred to the full version of this paper.

Theorem 1. Let Code $=$ (Init, Encode, Decode) be as above. Assume that $\left(\mathrm{LRS}, \mathrm{LRS}^{-1}\right)$ is an $\ell^{\prime}-L R S, \mathcal{H}=\left\{H_{t}:\{0,1\}^{\text {poly }(k)} \rightarrow\{0,1\}^{k}\right\}_{t \in\{0,1\}^{k}}$ is a family of collision resistant hash functions and $\left(\mathrm{G}_{\mathrm{NIZK}}\right.$, Prove, Verify) is a robust NIZK proof system for language $\mathcal{L}_{\mathcal{H}, t}$. Then Code is $(\ell, q)-C N M L R$, for any $q=\operatorname{poly}(k)$ and $\ell^{\prime} \geq \max \{2 \ell+(k+1)\lceil\log (q)\rceil, 2 k+1\}$.

\subsection{Outline of the Proof}

In order to build some intuition, let us first explain why a few natural attacks do not work. Clearly, the uniqueness property (cf. Lemma 3) rules out the attack of Lemma 1] As a first attempt, the adversary could try to modify the proof $\pi_{0}$ to a different proof $\pi_{0}^{\prime}$, by using the fact that $X_{0}$ contains the corresponding witness $s_{0}$ and the correct label $h_{1}$. However, to ensure the validity of $\left(X_{0}^{\prime}, X_{1}^{\prime}\right)$, this would require to place $\pi_{0}^{\prime}$ in $X_{1}^{\prime}$, which should be hard without knowing a witness (by the robustness of the proof system). Alternatively, one could try to maul the two halves $\left(s_{0}, s_{1}\right)$ of the LRS scheme, into a pair $\left(s_{0}^{\prime}, s_{1}^{\prime}\right)$ encoding a related message 6 This requires, for instance, to change the proof $\pi_{0}$ into $\pi_{0}^{\prime}$ and place $\pi_{0}^{\prime}$ in $X_{1}^{\prime}$, which again should be hard without knowing a witness and the correct label.

Let us now try to give a high-level overview of the proof. Given a polynomial time distinguisher $\mathrm{D}$ that violates continuous non-malleability of Code, we build another polynomial time distinguisher $\mathrm{D}^{\prime}$ which breaks leakage resilience of $\left(\mathrm{LRS}_{\mathrm{LRS}}^{-1}\right)$. Distinguisher $\mathrm{D}^{\prime}$, which can access oracles $\mathcal{O}^{\ell^{\prime}}\left(s_{0}\right)$ and $\mathcal{O}^{\ell^{\prime}}\left(s_{1}\right)$, has to distinguish whether $\left(s_{0}, s_{1}\right)$ is the encoding of message $x$ or message $y$ and will do so with the help of D's advantage in distinguishing $\operatorname{Tamper}_{x}^{\mathrm{cnmlr}}$ from Tamper $_{y}^{\mathrm{cnmlr}}$. The main difficulty in the reduction is how $\mathrm{D}^{\prime}$ can simulate the answers from the tampering oracle $\mathcal{O}_{\mathrm{cnm}}$ (cf. Definition 3), without knowing the target encoding $\left(X_{0}, X_{1}\right)$. This is the main point where our techniques diverge

${ }^{6}$ When the LRS is implemented using the inner product extractor this is indeed possible, as argued in [15. 
significantly from 24 (as in 24 the reduction "knows" a complete half of the encoding). In our case, in fact, $\mathrm{D}^{\prime}$ can only access the two halves $X_{0}$ and $X_{1}$ "inside" the oracles $\mathcal{O}^{\ell^{\prime}}\left(s_{0}\right)$ and $\mathcal{O}^{\ell^{\prime}}\left(s_{1}\right) 7$ However, it is not clear how this helps answering tampering queries, as the latter requires access to both $X_{0}$ and $X_{1}$ for decoding the tampered message, whereas the reduction can only access $X_{0}$ and $X_{1}$ separately.

For ease of description, in what follows we simply assume that $\mathrm{D}^{\prime}$ can access directly $\mathcal{O}^{\ell^{\prime}}\left(X_{0}\right)$ and $\mathcal{O}^{\ell^{\prime}}\left(X_{1}\right)$. Furthermore, let us assume that $\mathrm{D}$ can only issue tampering queries (we discuss how to additionally handle leakage briefly at the end of the outline). Like any standard reduction, $\mathrm{D}^{\prime}$ samples some randomness $r$ and fixes the random tape of $\mathbf{D}$ to $r$. Our novel strategy is to construct a polynomial time algorithm $\mathrm{F}(r)$ that, given access to $\mathcal{O}^{\ell^{\prime}}\left(X_{0}\right), \mathcal{O}^{\ell^{\prime}}\left(X_{1}\right)$, outputs the smallest index $j^{*}$ which indicates the round where $\mathrm{D}(r)$ provokes a selfdestruct in Tamper ${ }_{*}^{\text {cnmlr }}$. Before explaining how the actual algorithm works, let us explain how $\mathrm{D}^{\prime}$ can complete the reduction using such a self-destruct finder $\mathrm{F}$. At the beginning, it runs $\mathrm{F}(r)$ in order to leak the index $j^{*}$. At this point $\mathrm{D}^{\prime}$ is done with leakage queries and asks to get $X_{0}$ (i.e., it chooses $\theta=0$ in Definition 1) 8 Given $X_{0}$, distinguisher $\mathrm{D}^{\prime}$ runs $\mathrm{D}(r)$ (with the same random coins $r$ used for $\mathrm{F}$ ). Hence, for all $1 \leq j<j^{*}$, upon input the $j$-th tampering query $\left(\mathrm{T}_{0}^{(j)}, \mathrm{T}_{1}^{(j)}\right)$, distinguisher $\mathrm{D}^{\prime}$ lets $X_{0}^{\prime}=\mathrm{T}_{0}^{(j)}\left(X_{0}\right)=\left(s_{0}^{\prime}, h_{1}^{\prime}, \pi_{1}^{\prime}, \pi_{0}^{\prime}\right)$ and answers as follows:

1. In case $X_{0}^{\prime}=X_{0}$ (so called type A queries), output same*.

2. In case $X_{0}^{\prime} \neq X_{0}$ and either of the proofs in $X_{0}^{\prime}$ does not verify correctly (so called type B queries), output $\perp$.

3 . In case $X_{0}^{\prime} \neq X_{0}$ and both the proofs in $X_{0}^{\prime}$ verify correctly (so called type $\mathrm{C}$ queries), check if $\pi_{1}^{\prime}=\pi_{1}$; if 'yes' (in which case there is no hope to extract from $\pi_{1}^{\prime}$ ) then output $\perp$.

4. Otherwise (so called type $\mathrm{D}$ queries), attempt to extract $s_{1}^{\prime}$ from $\pi_{1}^{\prime}$, define $X_{1}^{\prime}=\left(s_{1}^{\prime}, h_{0}^{\prime}, \pi_{0}^{\prime}, \pi_{1}^{\prime}\right)$ and output $\left(X_{0}^{\prime}, X_{1}^{\prime}\right)$.

Note that from round $j^{*}$ on, all queries can be answered with $\perp$, and this is a correct simulation as $\mathrm{D}(r)$ provokes a self-destruct at round $j^{*}$ in the real experiment.

In the proof of Theorem 1, we show that the above strategy is sound. with overwhelming probability over the choice of $r$ the output produced by the above simulation is equal to the output that $\mathrm{D}(r)$ would have seen in the real experiment until a self-destruct occurs? 9

Let us give some intuition why the above simulation is indeed sound. For type A queries, note that when $X_{0}^{\prime}=X_{0}$ we must have $X_{1}^{\prime}=X_{1}$ with overwhelming

${ }^{7}$ Looking ahead, this can be achieved by first leaking the hash values $h_{0}, h_{1}$ of $s_{0}$, $s_{1}$, simulating the proofs $\pi_{0}, \pi_{1}$, and then hard-wiring these values into all leakage queries.

${ }^{8}$ Recall that this is a simplification, as by choosing $\theta=0$ the distinguisher will obtain $s_{0}$. See also footnote 7

${ }^{9}$ It is crucial that both the real and simulated experiments are run with the same $r$. 
probability, as otherwise $\left(X_{0}, X_{1}, X_{1}^{\prime}\right)$ would violate uniqueness. In case of type B queries, the decoding process in the real experiment would output $\perp$, so $D^{\prime}$ does a perfect simulation. The case of type $\mathrm{C}$ queries is a bit more delicate. In this case we use the facts that (i) in the NIZK proof system we use, different statements must have different proofs and (ii) the hash function is collision resistant, to show that $X_{0}^{\prime}$ must be of the form $X_{0}^{\prime}=\left(s_{0}, h_{1}, \pi_{1}, \pi_{0}^{\prime}\right)$ and $\pi_{0}^{\prime} \neq \pi_{1}$. A careful analysis shows that the latter contradicts leakage resilience of the underlying LRS scheme. Finally, for type D queries, note that whenever $\mathrm{D}^{\prime}$ extracts a witness from a valid proof $\pi_{1}^{\prime} \neq \pi_{1}$, the witness must be valid with overwhelming probability (as the NIZK is simulation extractable).

Next, let us explain how to construct the algorithm F. Roughly, F $r$ ) runs $\mathrm{D}(r)$ "inside" the oracles $\mathcal{O}^{\ell^{\prime}}\left(X_{0}\right), \mathcal{O}^{\ell^{\prime}}\left(X_{1}\right)$ as part of the leakage functions, and simulates the answers for $\mathrm{D}(r)$ 's tampering queries using only one side of the target encoding, in the exact same way as outlined in (1)-(4) above. Let $\boldsymbol{\Theta}_{b}$, for $b \in\{0,1\}$, denote the output simulated by $\mathrm{F}$ inside $\mathcal{O}^{\ell^{\prime}}\left(X_{b}\right)$. To locate the self-destruct index $j^{*}$, we rely on the following property: the vectors $\Theta_{0}$ and $\boldsymbol{\Theta}_{1}$ contain identical values until coordinate $j^{*}-1$, but $\boldsymbol{\Theta}_{0}\left[j^{*}\right] \neq \boldsymbol{\Theta}_{1}\left[j^{*}\right]$ with overwhelming probability (over the choice of $r$ ).

This implies that $j^{*}$ can be computed as the first coordinate where $\boldsymbol{\Theta}_{0}$ and $\boldsymbol{\Theta}_{1}$ are different. Hence, $F$ can obtain the self-destruct index by using its adaptive access to oracles $\mathcal{O}^{\ell^{\prime}}\left(X_{0}\right), \mathcal{O}^{\ell^{\prime}}\left(X_{1}\right)$ and apply a standard binary search algorithm to $\boldsymbol{\Theta}_{0}, \boldsymbol{\Theta}_{1}$. Note that the latter requires at most a logarithmic number of bits of adaptive leakage.

One technical problem is that $\mathrm{F}$, in order to run $\mathrm{D}(r)$ inside of, say $\mathcal{O}^{\ell^{\prime}}\left(X_{0}\right)$, and compute $\boldsymbol{\Theta}_{0}$, has also to answer leakage queries from $\mathrm{D}(r)$. Clearly, all leakages from $X_{0}$ can be easily computed, however it is not clear how to simulate leakages from $X_{1}$ (as we cannot access $\mathcal{O}^{\ell^{\prime}}\left(X_{1}\right)$ inside $\mathcal{O}^{\ell^{\prime}}\left(X_{0}\right)$ ). Fortunately, the latter issue can be avoided by letting $\mathrm{F}$ query $\mathcal{O}^{\ell^{\prime}}\left(X_{0}\right)$ and $\mathcal{O}^{\ell^{\prime}}\left(X_{1}\right)$ alternately, and aborting the execution of $\mathrm{D}(r)$ whenever it is not possible to answer a leakage query. It is not hard to show that after at most $\ell$ steps all leakages will be known, and $\mathrm{F}$ can run $\mathrm{D}(r)$ inside $\mathcal{O}^{\ell^{\prime}}\left(X_{0}\right)$ without having access to $\mathcal{O}^{\ell^{\prime}}\left(X_{1}\right)$. (All this comes at the price of some loss in the leakage bound, but, as we show in the proof, not too much.)

\section{Application to Tamper Resilient Security}

In this section we apply our notion of CNMLR codes to protect arbitrary functionalities against split-state tampering and leakage attacks.

\subsection{Stateless Functionalities}

We start by looking at the case of stateless functionalities $\mathcal{G}(s t, \cdot)$, which take as input a secret state $s t \in\{0,1\}^{k}$ and a value $x \in\{0,1\}^{u}$ to produce some output $y \in\{0,1\}^{v}$. The function $\mathcal{G}$ is public and can be randomized. 
The main idea is to transform the original functionality $\mathcal{G}(s t, \cdot)$ into some "hardened" functionality $\mathcal{G}^{\text {Code }}$ via a CNMLR code Code. Previous transformations aiming to protect stateless functionalities [17/24 required to freshly reencode the state st each time the functionality is invoked. Our approach avoids the re-encoding of the state at each invocation, leading to a stateless transformation. This solves an open question from [17. Moreover we consider a setting where the encoded state is stored in a memory $\left(\mathcal{M}_{0}, \mathcal{M}_{1}\right)$ which is much larger than the size needed to store the encoding itself (say $\left|\mathcal{M}_{0}\right|=\left|\mathcal{M}_{1}\right|=s$ where $s$ is polynomial in the length of the encoding). When (perfect) erasures are not possible, this feature allows the adversary to make copies of the initial encoding and tamper continuously with it, and was not considered in previous models.

Let us formally define what it means to harden a stateless functionality.

Definition 5 (Stateless hardened functionality). Let Code = (Init, Encode, Decode) be a split-state encoding scheme in the CRS model, with $k$ bits messages and $2 n$ bits codewords. Let $\mathcal{G}:\{0,1\}^{k} \times\{0,1\}^{u} \rightarrow\{0,1\}^{v}$ be a stateless functionality with secret state st $\in\{0,1\}^{k}$, and let $\varphi \in\{0,1\}$ be a public value initially set to zero. We define a stateless hardened functionality $\mathcal{G}^{\text {Code }}:\{0,1\}^{2 s} \times\{0,1\}^{u} \rightarrow\{0,1\}^{v}$ with a modified state st $\in\{0,1\}^{2 s}$ and $s=\operatorname{poly}(n)$. The hardened functionality $\mathcal{G}^{\text {Code }}$ is a triple of algorithms (Init, Setup, Execute) described as follows:

$-\Omega \leftarrow \operatorname{Init}\left(1^{k}\right):$ Run the initialization procedure of the coding scheme to sample $\Omega \leftarrow \operatorname{Init}\left(1^{k}\right)$.

$-\left(\mathcal{M}_{0}, \mathcal{M}_{1}\right) \leftarrow \operatorname{Setup}(\Omega, s t):$ Let $\left(X_{0}, X_{1}\right) \leftarrow \operatorname{Encode}(\Omega, s t)$. For $b \in\{0,1\}$, store $X_{b}$ in the first $n$ bits of $\mathcal{M}_{b}$, i.e. $\mathcal{M}_{b}[1 \ldots n] \leftarrow X_{b}$. (The remaining bits of $\mathcal{M}_{b}$ are set to $0^{s-n}$.) Define st' $:=\left(\mathcal{M}_{0}, \mathcal{M}_{1}\right)$.

$-y \leftarrow \operatorname{Execute}(x):$ Read the public value $\varphi$. In case $\varphi=1$ output $\perp$. Otherwise, let $X_{b}=\mathcal{M}_{b}[1 \ldots n]$ for $b \in\{0,1\}$. Run st $\leftarrow \operatorname{Decode}\left(\Omega,\left(X_{0}, X_{1}\right)\right)$; if st $=$ $\perp$, then output $\perp$ and set $\varphi=1$. Otherwise output $y \leftarrow \mathcal{G}($ st, $x)$.

Remark 1 (On $\varphi)$. The public value $\varphi$ is just a way how to implement the "self-destruct" feature. An alternative approach would be to let the hardened functionality simply output a dummy value and overwrite $\left(\mathcal{M}_{0}, \mathcal{M}_{1}\right)$ with the all-zero string. As we insist on the hardened functionality being stateless, we use the first approach here.

Note that we assume that $\varphi$ is untamperable. It is easy to see that this is necessary, as an adversary tampering with $\varphi$ could always switch-off the selfdestruct feature and apply a variant of the attack from [21] to recover the secret state.

Similarly to [1724, security of $\mathcal{G}^{\text {Code }}$ is defined via the comparison of a real and an ideal experiment. The real experiment features an adversary A interacting with $\mathcal{G}^{\text {Code }}$; the adversary is allowed to honestly run the functionality on any chosen input, but also to modify the secret state and retrieve a bounded amount of information from it. The ideal experiment features a simulator $\mathrm{S}$; the simulator is given black-box access to the original functionality $\mathcal{G}$ and to the adversary $\mathrm{A}$, 
but is not allowed any tampering or leakage query. The two experiments are formally described below.

Experiment $\operatorname{REAL}_{\mathrm{A}}^{\mathcal{G}^{\text {Code }}\left(s t^{\prime}, \cdot\right)}(k)$. First $\Omega \leftarrow \operatorname{Init}\left(1^{k}\right)$ and $\left(\mathcal{M}_{0}, \mathcal{M}_{1}\right) \leftarrow$ $\operatorname{Setup}(\Omega, s t)$ are run and $\Omega$ is given to A. Then A can issue the following commands polynomially many times (in any order):

- $\left\langle\right.$ Leak, $\left.\left(\mathrm{L}_{0}^{(j)}, \mathrm{L}_{1}^{(j)}\right)\right\rangle$ : In response to the $j$-th leakage query, compute $\Lambda_{0}^{(j)} \leftarrow$ $\mathrm{L}_{0}^{(j)}\left(\mathcal{M}_{0}\right)$ and $\Lambda_{1}^{(j)} \leftarrow \mathrm{L}_{1}^{(j)}\left(\mathcal{M}_{1}\right)$ and output $\left(\Lambda_{0}^{(j)}, \Lambda_{1}^{(j)}\right)$.

- $\left\langle\right.$ Tamper, $\left.\left(\mathrm{T}_{0}^{(j)}, \mathrm{T}_{1}^{(j)}\right)\right\rangle$ : In response to the $j$-th tampering query, compute $\mathcal{M}_{0}^{\prime} \leftarrow \mathrm{T}_{0}^{(j)}\left(\mathcal{M}_{0}\right)$ and $\mathcal{M}_{1}^{\prime} \leftarrow \mathrm{T}_{1}^{(j)}\left(\mathcal{M}_{1}\right)$ and replace $\left(\mathcal{M}_{0}, \mathcal{M}_{1}\right)$ with $\left(\mathcal{M}_{0}^{\prime}, \mathcal{M}_{1}^{\prime}\right)$.

- $\left\langle\right.$ Eval, $\left.x_{j}\right\rangle$ : In response to the $j$-th evaluation query, run $y_{j} \leftarrow \operatorname{Execute}\left(x_{j}\right)$. In case $y_{j}=\perp$ output $\perp$ and self-destruct; otherwise output $y_{j}$.

The output of the experiment is defined as

$$
\operatorname{REAL}_{\mathrm{A}}^{\mathcal{G}^{\text {Code }}\left(s t^{\prime}, \cdot\right)}(k)=\left(\Omega ;\left(\left(x_{1}, y_{1}\right),\left(x_{2}, y_{2}\right), \ldots\right) ;\left(\left(\Lambda_{0}^{(1)}, \Lambda_{1}^{(1)}\right),\left(\Lambda_{0}^{(2)}, \Lambda_{1}^{(2)}\right), \cdots\right)\right)
$$

Experiment $\operatorname{IDEAL}_{\mathrm{S}}^{\mathcal{G}(s t, \cdot)}(k)$. The simulator sets up the CRS $\Omega$ and is given black-box access to the functionality $\mathcal{G}(s t, \cdot)$ and the adversary A. The output of the experiment is defined as

$$
\operatorname{IDEAL}_{\mathrm{S}}^{\mathcal{G}(s t, \cdot)}(k)=\left(\Omega ;\left(\left(x_{1}, y_{1}\right),\left(x_{2}, y_{2}\right), \ldots\right) ;\left(\left(\Lambda_{0}^{(1)}, \Lambda_{1}^{(1)}\right),\left(\Lambda_{0}^{(2)}, \Lambda_{1}^{(2)}\right), \cdots\right)\right),
$$

where $\left(\left(x_{j}, y_{j}\right),\left(\left(\Lambda_{0}^{(j)}, \Lambda_{1}^{(j)}\right)\right)\right)$ are the input/output/leakage tuples simulated by S.

Definition 6 (Polyspace leak/tamper simulatability). Let Code be a splitstate encoding scheme in the CRS model and consider a stateless functionality $\mathcal{G}$ with corresponding hardened functionality $\mathcal{G}^{\text {Code }}$. We say that Code is polyspace $(\ell, q)$-leak/tamper simulatable for $\mathcal{G}$, if the following conditions are satisfied:

1. Each memory part $\mathcal{M}_{b}($ for $b \in\{0,1\})$ has size $s=\operatorname{poly}(n)$.

2. The adversary asks at most $q$ tampering queries and leaks a total of at most $\ell$ bits from each memory part.

3. For all PPT adversaries A there exists a PPT simulator $\mathrm{S}$ such that for any initial state st,

$$
\left\{\operatorname{REAL}_{\mathrm{A}}^{\mathcal{G}^{\text {Code }}\left(s t^{\prime}, \cdot\right)}(k)\right\}_{k \in \mathbb{N}} \approx_{c}\left\{\operatorname{IDEAL}_{\mathrm{S}}^{\mathcal{G}(s t, \cdot)}(k)\right\}_{k \in \mathbb{N}}
$$

We show the following result.

Theorem 2. Let $\mathcal{G}$ be a stateless functionality and Code = (Init, Encode, Decode) be any $(\ell, q)-C N M L R$ split-state encoding scheme in the CRS model. Then Code is polyspace $(\ell, q)$-leak/tamper simulatable for $\mathcal{G}$. 
Proof. We discuss the overall proof approach first. We start with describing a simulator S running in experiment $\operatorname{IDEAL}_{\mathrm{S}}^{\mathcal{G}(s t, \cdot)}(k)$ which attempts to simulate the view of adversary $A$ running in the experiment $\operatorname{REAL}_{\mathrm{A}}^{\mathcal{G}^{\text {Code }}\left(s t^{\prime}, \cdot\right)}(k)$; the simulator is given black-box access to $A$ (which can issue Tamper, Leak, and Eval queries) and to the functionality $\mathcal{G}(s t, \cdot)$ for some state st. To argue that our simulator is "good" we show that if there exists a PPT distinguisher D and a PPT adversary A such that for some state st, D distinguishes the experiments $\operatorname{IDEAL}_{\mathrm{S}}^{\mathcal{G}(s t, \cdot)}(k)$ and $\operatorname{REAL}_{\mathrm{A}}^{\mathcal{G}^{\text {Code }}\left(s t^{\prime}, \cdot\right)}(k)$ with non-negligible probability, then we can build another distinguisher $D^{\prime}$ and an adversary $A^{\prime}$ such that $D^{\prime}$ can distinguish $\operatorname{Tamper}_{\mathrm{A}^{\prime}, 0^{k}}^{\mathrm{cnmlr}}$ and $\operatorname{Tamper}_{\mathrm{A}^{\prime}, s t}^{\mathrm{cnmlr}}$ with non-negligible probability. In the last step essentially we reduce the CNMLR property of Code to the polyspace leak/tamper simulatability of the code itself.

The simulator starts by sampling the common reference string $\Omega \leftarrow \operatorname{Init}\left(1^{k}\right)$ and the public value $\varphi=0$. Then it samples a random encoding of $0^{k}$, namely $\left(Z_{0}, Z_{1}\right) \leftarrow \operatorname{Encode}\left(\Omega, 0^{k}\right)$ and sets $\mathcal{M}_{b}[1 \ldots, n] \leftarrow Z_{b}$ for $b \in\{0,1\}$. The remaining bits of $\left(\mathcal{M}_{0}, \mathcal{M}_{1}\right)$ are set to $0^{s-n}$. Hence, $S$ alternates between the following two modes (starting with the normal mode in the first round):

- Normal Mode. Given state $\left(\mathcal{M}_{0}, \mathcal{M}_{1}\right)$, while A continues issuing queries, answer as follows:

- $\left\langle\right.$ Eval, $\left.x_{j}\right\rangle$ : Upon input the $j$-th evaluation query invoke $\mathcal{G}(s t, \cdot)$ to get $y_{j} \leftarrow \mathcal{G}\left(s t, x_{j}\right)$ and reply with $y_{j}$.

- 〈Tamper, $\left.\left(\mathrm{T}_{0}^{(j)}, \mathrm{T}_{1}^{(j)}\right)\right\rangle$ : Upon input the $j$-th tampering query, compute $\mathcal{M}_{b}^{\prime} \leftarrow \mathrm{T}_{b}^{(j)}\left(\mathcal{M}_{b}\right)$ for $b \in\{0,1\}$. In case $\left(\mathcal{M}_{0}^{\prime}[1 \ldots n], \mathcal{M}_{1}^{\prime}[1 \ldots n]\right)=$ $\left(Z_{0}, Z_{1}\right)$ then continue in the current mode. Otherwise go to the overwritten mode defined below with state $\left(\mathcal{M}_{0}^{\prime}, \mathcal{M}_{1}^{\prime}\right)$.

- $\left\langle\right.$ Leak, $\left.\left(\mathrm{L}_{0}^{(j)}, \mathrm{L}_{1}^{(j)}\right)\right\rangle$ : Upon input the $j$-th leakage query, compute $\Lambda_{b}^{(j)}=$ $\mathrm{L}_{b}^{(j)}\left(Z_{b}\right)$ for $b \in\{0,1\}$ and reply with $\left(\Lambda_{0}^{(j)}, \Lambda_{1}^{(j)}\right)$.

- Overwritten Mode. Given state $\left(\mathcal{M}_{0}^{\prime}, \mathcal{M}_{1}^{\prime}\right)$, while A continues issuing queries, answer as follows:

- Let $\tau=\left(\mathcal{M}_{0}^{\prime}, \mathcal{M}_{1}^{\prime}\right)$. Simulate the hardened functionality $\mathcal{G}^{\text {Code }}(\tau, \cdot)$ and answer all Eval and Leak queries as the real experiment $\operatorname{REAL}_{\mathrm{A}}^{\mathcal{G}^{\text {Code }}(\tau, \cdot)}(k)$ would do.

- Upon input the $j$-th tampering query $\left(\mathrm{T}_{0}^{(j)}, \mathrm{T}_{1}^{(j)}\right)$, compute $\mathcal{M}_{b}^{\prime \prime} \leftarrow$ $\mathrm{T}_{b}^{(j)}\left(\mathcal{M}_{b}^{\prime}\right)$ for $b \in\{0,1\}$. In case $\left(\mathcal{M}_{0}^{\prime \prime}[1 \ldots n], \mathcal{M}_{1}^{\prime \prime}[1 \ldots n]\right)=\left(Z_{0}, Z_{1}\right)$ then go to the normal mode with state $\left(\mathcal{M}_{0}, \mathcal{M}_{1}\right):=\left(\mathcal{M}_{0}^{\prime \prime}, \mathcal{M}_{1}^{\prime \prime}\right)$. Otherwise continue in the current mode.

- When A halts and outputs view $=\left(\Omega ;\left(\left(x_{1}, y_{1}\right),\left(x_{2}, y_{2}\right), \ldots\right)\right.$; $\left.\left(\left(\Lambda_{0}^{(1)}, \Lambda_{1}^{(1)}\right),\left(\Lambda_{0}^{(2)}, \Lambda_{1}^{(2)}\right), \cdots\right)\right)$, set views $=$ view $_{\mathrm{A}}$ and output views as output of $\operatorname{IDEAL}_{\mathrm{S}}^{\mathcal{G}(s t, \cdot)}(k)$.

Intuitively, since the coding scheme is non-malleable, the adversary can either keep the encoding unchanged or overwrite it with the encoding of some unrelated message. These two cases are captured in the above modes: The simulator starts 
in the normal mode and then, whenever the adversary mauls the initial encoding, it switches to the overwritten mode. However, the adversary can use the extra space to keep a copy of the original encoding and place it back at some later point in time. When this happens, the simulator switches back to the normal mode; this switching is important to maintain simulation.

To finish the proof, we have to argue that the output of experiment $\operatorname{IDEAL}_{\mathrm{S}}^{\mathcal{G}(s t, \cdot)}(k)$ is computationally indistinguishable from the output of experiment $\operatorname{REAL}_{\mathrm{A}}^{\mathcal{G}^{\text {Code }}\left(s t^{\prime}, \cdot\right)}(k)$. This is done in the lemma below.

Lemma 4. Let $\mathrm{S}$ be defined as above. Then for all PPT adversaries A and all st $\in\{0,1\}^{k}$, the following holds:

$$
\left\{\operatorname{REAL}_{\mathrm{A}}^{\mathcal{G}^{\text {Code }}\left(s t^{\prime}, \cdot\right)}(k)\right\}_{k \in \mathbb{N}} \approx_{c}\left\{\operatorname{IDEAL}_{\mathrm{S}}^{\mathcal{G}(s t, \cdot)}(k)\right\}_{k \in \mathbb{N}}
$$

Proof. By contradiction, assume that there exists a PPT distinguisher D, a PPT adversary $\mathrm{A}$ and some state $s t \in\{0,1\}^{k}$ such that:

$$
\left|\mathbb{P}\left[\mathrm{D}\left(\operatorname{IDEAL}_{\mathrm{S}}^{\mathcal{G}(s t, \cdot)}(k)\right)=1\right]-\mathbb{P}\left[\mathrm{D}\left(\operatorname{REAL}_{\mathrm{A}}^{\mathcal{G}^{\text {Code }}\left(s t^{\prime}, \cdot\right)}(k)\right)=1\right]\right| \geq \epsilon,
$$

where $\epsilon(k)$ is some non-negligible function of the security parameter $k$.

We build a PPT distinguisher $\mathrm{D}^{\prime}$ and a PPT adversary $\mathrm{A}^{\prime}$ telling apart the experiments $\operatorname{Tamper}_{\mathrm{A}^{\prime}, 0^{k}}^{\mathrm{cnmlr}}(k)$ and $\operatorname{Tamper}_{\mathrm{A}^{\prime}, s t}^{\mathrm{cnmlr}}(k)$; this contradicts our assumption that Code is CNMLR. The distinguisher $\mathrm{D}^{\prime}$ is given the CRS $\Omega \leftarrow \operatorname{Init}\left(1^{k}\right)$ and can access $\mathcal{O}_{\mathrm{cnm}}\left(\left(X_{0}, X_{1}\right), \cdot\right)$ (for at most $q$ times) and $\mathcal{O}^{\ell}\left(X_{0}\right), \mathcal{O}^{\ell}\left(X_{1}\right)$; here $\left(X_{0}, X_{1}\right)$ is either an encoding of $0^{k}$ or an encoding of st. The distinguisher $\mathrm{D}^{\prime}$ keeps a flag SAme (initially set to TRUE) and a flag STOP (initially set to FALSE). After simulating the public values, $D^{\prime}$ mimics the enviroment for D as follows:

- $\left\langle\right.$ Tamper, $\left.\left(\mathrm{T}_{0}^{(j)}, \mathrm{T}_{1}^{(j)}\right)\right\rangle$ : Upon input tampering functions $\left(\mathrm{T}_{0}^{(j)}, \mathrm{T}_{1}^{(j)}\right)$, the distinguisher $\mathrm{D}^{\prime}$ uses the oracle $\mathcal{O}_{\mathrm{cnm}}\left(\left(X_{0}, X_{1}\right), \cdot\right)$ to answer them 10 However, it can not simply forward the queries because of the following two reasons:

- The tampering functions $\left(\mathrm{T}_{0}^{(j)}, \mathrm{T}_{1}^{(j)}\right)$ maps from $s$ bits to $s$ bits, whereas the oracle $\mathcal{O}_{\mathrm{cnm}}\left(\left(X_{0}, X_{1}\right), \cdot\right)$ expects tampering functions mapping from $n$ bits to $n$ bits.

- In both the real and the ideal experiments the tampering functions are applied to the current state (which may be different from the initial state), whereas in experiment $\operatorname{Tamper}_{\mathrm{A}^{\prime}, *}^{\mathrm{cnmlr}}$ the oracle $\mathcal{O}_{\mathrm{cnm}}\left(\left(X_{0}, X_{1}\right), \cdot\right)$ always applies $\left(\mathrm{T}_{0}^{(j)}, \mathrm{T}_{1}^{(j)}\right)$ to the target encoding $\left(X_{0}, X_{1}\right)$.

To take into account the above differences, $\mathrm{D}^{\prime}$ modifies $\left(\mathrm{T}_{0}^{(j)}, \mathrm{T}_{1}^{(j)}\right)$ as follows. Define the functions $\mathrm{T}_{\text {in }}:\{0,1\}^{n} \rightarrow\{0,1\}^{s}$ and $\mathrm{T}_{\text {out }}:\{0,1\}^{s} \rightarrow\{0,1\}^{n}$ as $\mathrm{T}_{\text {in }}(x)=\left(x \| 0^{s-n}\right)$ and $\mathrm{T}_{\text {out }}\left(x \| x^{\prime}\right)=x$, for any $x \in\{0,1\}^{n}$ and $x^{\prime} \in$ $\{0,1\}^{s-n}$. The distinguisher $\mathrm{D}^{\prime}$ queries $\mathcal{O}_{\mathrm{cnm}}\left(\left(X_{0}, X_{1}\right), \cdot\right)$ with the function

$\overline{{ }^{10} \text { Formally } \mathrm{D}^{\prime}}$ has to access $\mathcal{O}_{\mathrm{cnm}}(\cdot)$ via $\mathrm{A}^{\prime}$. For simplicity we assume that $\mathrm{D}^{\prime}$ can access the oracle directly. In fact, $A^{\prime}$ just acts as an interface between the experiment

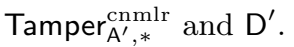


pair $\left(\tilde{\mathbf{T}}_{0}^{(j)}, \tilde{\mathbf{T}}_{1}^{(j)}\right)$ where each $\tilde{\mathbf{T}}_{b}^{(j)}$ is defined as $\tilde{\mathrm{T}}_{b}^{(j)}:=\mathrm{T}_{\text {out }} \circ \mathrm{T}_{b}^{(j)} \circ \mathrm{T}_{b}^{(j-1)} \circ$ $\ldots \circ \mathrm{T}_{b}^{(1)} \circ \mathrm{T}_{\text {in }}$ for $b \in\{0,1\}$.

In case the oracle returns $\perp$, then $\mathrm{D}^{\prime}$ sets Stop to True. In case the oracle returns same ${ }^{\star}$, then $\mathrm{D}^{\prime}$ sets SAmE to True. Otherwise, in case the oracle returns an encoding $\left(X_{0}^{\prime}, X_{1}^{\prime}\right)$, then $\mathrm{D}^{\prime}$ sets SAME to FALSE.

- $\left\langle\right.$ Leak, $\left.\left(\mathrm{L}_{0}^{(j)}, \mathrm{L}_{1}^{(j)}\right)\right\rangle$ : Upon input leakage functions $\left(\mathrm{L}_{0}^{(j)}, \mathrm{L}_{1}^{(j)}\right)$, the distinguisher $\mathrm{D}^{\prime}$ defines $\left(\tilde{\mathrm{L}}_{0}^{(j)}, \tilde{\mathrm{L}}_{1}^{(j)}\right.$ ) (in a similar way as above), forwards those functions to $\mathcal{O}^{\ell}\left(X_{0}\right), \mathcal{O}^{\ell}\left(X_{1}\right)$ and sends the answer from the oracles back to D.

- $\left\langle\right.$ Eval, $\left.x_{j}\right\rangle$ : Upon input an evaluation query for value $x_{j}$, the distinguisher $\mathrm{D}^{\prime}$ checks first that STOP equals FALSE. If this is not the case, then $\mathrm{D}^{\prime}$ returns $\perp$ to $D$. Otherwise, $D^{\prime}$ checks that SAmE equals True. If this is the case, it runs $y_{j} \leftarrow \mathcal{G}\left(s t, x_{j}\right)$ and gives $y_{j}$ to D. Else (if SAmE equals FALSE), it computes $y_{j} \leftarrow \mathcal{G}\left(s t^{\prime}, x_{j}\right)$, where $s t^{\prime}$ is the output of $\operatorname{Decode}\left(\Omega,\left(X_{0}^{\prime}, X_{1}^{\prime}\right)\right)$, and gives $y_{j}$ to $\mathrm{D}$.

Finally, D' outputs whatever D outputs.

For the analysis, first note that $\mathrm{D}^{\prime}$ runs in polynomial time. Furthermore, $\mathrm{D}^{\prime}$ asks exactly $q$ queries to $\mathcal{O}_{\mathrm{cnm}}$ and leaks at most $\ell$ bits from the target encoding $\left(X_{0}, X_{1}\right)$. It is also easy to see that in case $\left(X_{0}, X_{1}\right)$ is an encoding of st $\epsilon$ $\{0,1\}^{k}$, then $D^{\prime}$ perfectly simulates the view of adversary $\mathrm{D}$ in the experiment $\operatorname{REAL}_{\mathrm{A}}^{\mathcal{G}^{\text {Code }}\left(s t^{\prime}, \cdot\right)}(k)$. On the other hand, in case $\left(X_{0}, X_{1}\right)$ is an encoding of $0^{k}$, we claim that $\mathrm{D}^{\prime}$ perfectly simulates the view of $\mathrm{D}$ in the experiment $\operatorname{IDEAL}_{S}^{\mathcal{G}(s t, \cdot)}(k)$. This is because: (i) Whenever SAmE equals True, then $\mathrm{D}^{\prime}$ answers evaluation queries by running $\mathcal{G}$ on state $s t$ and tampering/leakage queries using a presampled encoding of $0^{k}$ (this corresponds to the normal mode of S); (ii) Whenever SAME equals FALSE, then $D^{\prime}$ answers evaluation queries by running $\mathcal{G}$ on the current tampered state $s t^{\prime}$ which results from applying the tampering functions to a pre-sampled encoding of $0^{k}$ (this corresponds to the overwritten mode of S).

Combining the above argument with Eq. (1) we obtain

$$
\left|\mathbb{P}\left[\mathrm{D}\left(\operatorname{Tamper}_{\mathrm{A}^{\prime}, 0^{k}}^{\mathrm{cnmml}}(k)\right)=1\right]-\mathbb{P}\left[\mathrm{D}\left(\operatorname{Tamper}_{\mathrm{A}^{\prime}, s t}^{\mathrm{cnmlr}}(k)\right)=1\right]\right| \geq \epsilon,
$$

which is a contradiction to the fact that Code is $(\ell, q)$-CNMLR.

\subsection{Stateful Functionalities}

Finally, we consider the case of primitives that update their state at each execution, i.e. functionalities of the type $\left(s t_{\text {new }}, y\right) \leftarrow \mathcal{G}(s t, x)$ (a.k.a. stateful functionalities). Note that in this case the hardened functionality re-encodes the new state at each execution.

Note that, since we do not assume erasure in our model, an adversary can always 'reset' the functionality to a previous valid state as follows: It could just copy the previous state to some part of the large memory and replace the current 
encoding by that. To avoid this, our transformation uses an untamperable public counter (along with the untamperable self-destruct bit) that helps us to detect whether the functionality is reset to a previous state, leading to a self-destruct. However such a counter can be implemented, for instance using $\log (k)$ bits. We notice that such a counter is necessary to protect against the above resetting attack. However, we stress that if we do not assume such a counter this "resetting" is the only harm the adversary can make in our model.

Below, we define what it means to harden a stateful functionality.

Definition 7 (Stateful hardened functionality). Let Code = (Init, Encode, Decode) be a split-state encoding scheme in the CRS model, with $2 k$ bits messages and $2 n$ bits codewords. Let $\mathcal{G}:\{0,1\}^{k} \times\{0,1\}^{u} \rightarrow$ $\{0,1\}^{k} \times\{0,1\}^{v}$ be a stateful functionality with secret state st $\in\{0,1\}^{k}$, $\varphi \in\{0,1\}$ be a public value and let $\langle\gamma\rangle$ be a public $\log (k)$-bit counter both initially set to zero. We define a stateful hardened functionality $\mathcal{G}^{\text {Code }}:\{0,1\}^{2 s} \times\{0,1\}^{u} \rightarrow\{0,1\}^{2 s} \times\{0,1\}^{v}$ with a modified state st $\in\{0,1\}^{2 s}$ and $s=\operatorname{poly}(n)$. The hardened functionality $\mathcal{G}^{\text {Code }}$ is a triple of algorithms (Init, Setup, Execute) described as follows:

$-\Omega \leftarrow \operatorname{Init}\left(1^{k}\right):$ Run the initialization procedure of the coding scheme to sample $\Omega \leftarrow \operatorname{Init}\left(1^{k}\right)$.

$-\left(\mathcal{M}_{0}, \mathcal{M}_{1}\right) \leftarrow \operatorname{Setup}(\Omega, s t):$ Let $\left(X_{0}, X_{1}\right) \leftarrow \operatorname{Encode}(\Omega, s t||\langle 1\rangle)$ and increment $\langle\gamma\rangle \leftarrow\langle\gamma\rangle+1$. For $b \in\{0,1\}$, store $X_{b}$ in the first $n$ bits of $\mathcal{M}_{b}$, i.e. $\mathcal{M}_{b}[1 \ldots n] \leftarrow X_{b} 11$ (The remaining bits of $\mathcal{M}_{b}$ are set to $0^{s-n}$.) Define $s t^{\prime}:=\left(\mathcal{M}_{0}, \mathcal{M}_{1}\right)$.

$-y \leftarrow \operatorname{Execute}(x):$ Read the public bit $\varphi$. In case $\varphi=1$ output $\perp$. Otherwise recover $X_{b}=\mathcal{M}_{b}[1 \ldots n]$ for $b \in\{0,1\}$ and run $\left(s t^{\prime \prime} \|\left\langle\gamma^{\prime}\right\rangle\right) \leftarrow$ $\operatorname{Decode}\left(\Omega,\left(X_{0}, X_{1}\right)\right)$. Read the public counter $\langle\gamma\rangle$. If $\langle\gamma\rangle \neq\left\langle\gamma^{\prime}\right\rangle$ or $s t^{\prime \prime}=$ $\perp$, set $\varphi=1$. Else run $\left(s_{\text {new }}, y\right) \leftarrow \mathcal{G}\left(s t^{\prime \prime}, x\right)$ and output $y$. Finally, write $\operatorname{Encode}\left(\Omega, s t_{\text {new }} \mid\langle\gamma+1\rangle\right)$ in $\left(\mathcal{M}_{0}[1, \ldots, n], \mathcal{M}_{1}[1, \ldots, n]\right)$ and increment $\langle\gamma\rangle \leftarrow\langle\gamma\rangle+1$.

Remark $2(O n\langle\gamma\rangle)$. Note that the counter is incremented after each evaluation query, and the current value is encoded together with the new state. We require $\langle\gamma\rangle$ to be untamperable. This assumption is necessary, as otherwise an adversary could always use the extra space to keep a copy of a previous valid state and place it back at some later point in time. The above attack allows essentially to reset the functionality to a previous state, and cannot be simulated with black-box access to the original functionality.

In the case of stateful primitives, the hardened functionality has to re-encode the new state at each execution. Still, as the memory is large, the adversary can use the extra space to tamper continuously with a target encoding of some valid state. Security of a stateful hardened functionality is defined analogously to the stateless case (cf. Definition 6). We show the following result (for space reasons we defer the proof to the full version [18]):

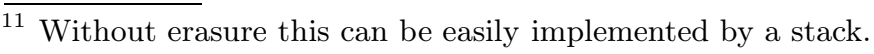


Theorem 3. Let $\mathcal{G}$ be a stateful functionality and Code = (Init, Encode, Decode) be any $(\ell, q)-C N M L R$ encoding scheme in the split-state CRS model. Then Code is polyspace $(\ell, q)$-leak/tamper simulatable for $\mathcal{G}$.

Acknowledgments. Pratyay acknowledges support from a European Research Commission Starting Grant (no. 279447) and the CTIC and CFEM research center. Part of this work was done while this author was at the University of Warsaw and was supported by the WELCOME/2010-4/2 grant founded within the framework of the EU Innovative Economy Operational Programme.

Most of the work was done while Daniele was at Aarhus University, supported by the Danish Council for Independent Research via DFF Starting Grant 10-081612.

\section{References}

1. Aggarwal, D., Dodis, Y., Lovett, S.: Non-malleable codes from additive combinatorics. Electronic Colloquium on Computational Complexity (ECCC) 20, 81 (2013)

2. Austrin, P., Chung, K.-M., Mahmoody, M., Pass, R., Seth, K.: On the (im)possibility of tamper-resilient cryptography: Using fourier analysis in computer viruses. IACR Cryptology ePrint Archive 2013, 194 (2013)

3. Bellare, M., Cash, D., Miller, R.: Cryptography secure against related-key attacks and tampering. In: Lee, D.H., Wang, X. (eds.) ASIACRYPT 2011. LNCS, vol. 7073, pp. 486-503. Springer, Heidelberg (2011)

4. Bellare, M., Kohno, T.: A theoretical treatment of related-key attacks: RKA-PRPs, RKA-PRFs, and applications. In: Biham, E. (ed.) EUROCRYPT 2003. LNCS, vol. 2656, pp. 491-506. Springer, Heidelberg (2003)

5. Bellare, M., Paterson, K.G., Thomson, S.: RKA security beyond the linear barrier: IBE, encryption and signatures. In: Wang, X., Sako, K. (eds.) ASIACRYPT 2012. LNCS, vol. 7658, pp. 331-348. Springer, Heidelberg (2012)

6. Boneh, D., DeMillo, R.A., Lipton, R.J.: On the importance of eliminating errors in cryptographic computations. J. Cryptology 14(2), 101-119 (2001)

7. Cheraghchi, M., Guruswami, V.: Capacity of non-malleable codes. Electronic Colloquium on Computational Complexity (ECCC) 20, 118 (2013)

8. Cheraghchi, M., Guruswami, V.: Non-malleable coding against bit-wise and splitstate tampering. IACR Cryptology ePrint Archive 2013, 565 (2013)

9. Choi, S.G., Kiayias, A., Malkin, T.: BiTR: Built-in tamper resilience. In: Lee, D.H., Wang, X. (eds.) ASIACRYPT 2011. LNCS, vol. 7073, pp. 740-758. Springer, Heidelberg (2011)

10. Chor, B., Goldreich, O.: Unbiased bits from sources of weak randomness and probabilistic communication complexity. SIAM J. Comput. 17(2), 230-261 (1988)

11. Dachman-Soled, D., Kalai, Y.T.: Securing circuits against constant-rate tampering. In: Safavi-Naini, R., Canetti, R. (eds.) CRYPTO 2012. LNCS, vol. 7417, pp. 533-551. Springer, Heidelberg (2012)

12. Damgård, I., Faust, S., Mukherjee, P., Venturi, D.: Bounded tamper resilience: How to go beyond the algebraic barrier. In: Sako, K., Sarkar, P. (eds.) ASIACRYPT 2013, Part II. LNCS, vol. 8270, pp. 140-160. Springer, Heidelberg (2013)

13. Davì, F., Dziembowski, S., Venturi, D.: Leakage-resilient storage. In: Garay, J.A., De Prisco, R. (eds.) SCN 2010. LNCS, vol. 6280, pp. 121-137. Springer, Heidelberg (2010) 
14. Dziembowski, S., Faust, S.: Leakage-resilient cryptography from the inner-product extractor. In: Lee, D.H., Wang, X. (eds.) ASIACRYPT 2011. LNCS, vol. 7073, pp. 702-721. Springer, Heidelberg (2011)

15. Dziembowski, S., Kazana, T., Obremski, M.: Non-malleable codes from two-source extractors. In: Canetti, R., Garay, J.A. (eds.) CRYPTO 2013, Part II. LNCS, vol. 8043, pp. 239-257. Springer, Heidelberg (2013)

16. Dziembowski, S., Pietrzak, K.: Leakage-resilient cryptography. In: FOCS, pp. 293-302 (2008)

17. Dziembowski, S., Pietrzak, K., Wichs, D.: Non-malleable codes. In: ICS, pp. 434-452 (2010)

18. Faust, S., Mukherjee, P., Nielsen, J.B., Venturi, D.: Continuous non-malleable codes (2013). The full version will be available at the IACR Cryptology ePrint Archive

19. Faust, S., Mukherjee, P., Venturi, D., Wichs, D.: Efficient non-malleable codes and key-derivation for poly-size tampering circuits. IACR Cryptology ePrint Archive 2013, 702 (2013)

20. Faust, S., Pietrzak, K., Venturi, D.: Tamper-proof circuits: How to trade leakage for tamper-resilience. In: Aceto, L., Henzinger, M., Sgall, J. (eds.) ICALP 2011, Part I. LNCS, vol. 6755, pp. 391-402. Springer, Heidelberg (2011)

21. Gennaro, R., Lysyanskaya, A., Malkin, T., Micali, S., Rabin, T.: Algorithmic tamper-proof (ATP) security: Theoretical foundations for security against hardware tampering. In: Naor, M. (ed.) TCC 2004. LNCS, vol. 2951, pp. 258-277. Springer, Heidelberg (2004)

22. Ishai, Y., Prabhakaran, M., Sahai, A., Wagner, D.: Private circuits II: Keeping secrets in tamperable circuits. In: Vaudenay, S. (ed.) EUROCRYPT 2006. LNCS, vol. 4004, pp. 308-327. Springer, Heidelberg (2006)

23. Kalai, Y.T., Kanukurthi, B., Sahai, A.: Cryptography with tamperable and leaky memory. In: Rogaway, P. (ed.) CRYPTO 2011. LNCS, vol. 6841, pp. 373-390. Springer, Heidelberg (2011)

24. Liu, F.-H., Lysyanskaya, A.: Tamper and leakage resilience in the split-state model. In: Safavi-Naini, R., Canetti, R. (eds.) CRYPTO 2012. LNCS, vol. 7417, pp. 517-532. Springer, Heidelberg (2012)

25. Pietrzak, K.: Subspace lwe. In: Cramer, R. (ed.) TCC 2012. LNCS, vol. 7194, pp. 548-563. Springer, Heidelberg (2012)

26. De Santis, A., Di Crescenzo, G., Ostrovsky, R., Persiano, G., Sahai, A.: Robust noninteractive zero knowledge. In: Kilian, J. (ed.) CRYPTO 2001. LNCS, vol. 2139, pp. 566-598. Springer, Heidelberg (2001) 